\title{
Disparities in fresh fruit and vegetable intake by sociodemographic and behavioural factors among adults in China
}

\author{
Li Li, Yifei Ouyang, Huijun Wang, Feifei Huang, Yun Wang, Jiguo Zhang, Chang Su, \\ Wenwen Du, Xiaofang Jia, Hongru Jiang, Zhihong Wang and Bing Zhang* \\ National Institute for Nutrition and Health, Chinese Center for Disease Control and Prevention, 27 Nanwei Rd, Xicheng \\ District, Beijing 100050, People's Republic of China
}

Submitted 7 January 2020: Final revision received 2 July 2020: Accepted 14 August 2020: First published online 14 September 2020

\begin{abstract}
Objective: To measure the associations of sociodemographic and behavioural factors with fruit and vegetable consumption among adults in China.

Design: A cross-sectional study.

Setting: A 2015 wave of the China Health and Nutrition Survey.

Participants: Totally, 11910 adults aged 18 to 64 years.

Results: Adjusted log binomial regression analyses showed that adults with higher income levels had higher fruit intake than those with low income levels (medium income group, risk ratio (RR): 1.28; $95 \%$ CI: 1·16, 1.41; high income group, RR: 1.58; $95 \%$ CI: 1.43, 1.74). Current smokers had lower fruit intake than non-smokers (RR: $0 \cdot 86 ; 95 \%$ CI: $0 \cdot 77,0.96)$. Adults living in southern China had higher vegetable intake (RR: 1.88; $95 \%$ CI: 1.76, 2.01) but lower fruit intake (RR: 0.85; $95 \%$ CI: 0.79, 0.91) than adults in northern China. With increasing age, adults had higher fruit intake (50-64 years, RR: 1.20; $95 \%$ CI: 1.09, 1.33; reference category 18-34 years) and higher vegetable intake (35-49 years, RR: 1·13; $95 \%$ CI: 1·05, 1·22; 50-64 years, RR: $1 \cdot 22$; $95 \%$ CI: $1 \cdot 13,1 \cdot 31$ ).

Conclusions: Our findings identify a range of sociodemographic and behavioural factors associated with fruit and vegetable consumption among Chinese adults. They also point to the need for public health nutrition interventions for socially disadvantaged populations in China.
\end{abstract}

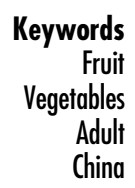

Fruits and vegetables ( $\mathrm{F} \& \mathrm{~V}$ ) are important sources of nutrients, dietary fibre and several classes of phytochemicals and are instrumental in disease prevention ${ }^{(1)}$. Studies show that intake of fruits and vegetables is inversely associated with the risk of cancer, heart disease, diabetes, stroke and obesity ${ }^{(2-9)}$. A systematic analysis found that low fruit intake is one of the leading dietary risk factors for death and reduction in disability-adjusted life years in many countries ${ }^{(10)}$. The WHO and the World Cancer Research Fund both recommend fruit and vegetable intake of at least $400 \mathrm{~g} / \mathrm{d}^{(1)}$. These recommendations are based on studies reporting the fruit and vegetable consumption levels that are associated with reduced risk of disease.

The 2016 Dietary Guidelines for Chinese Residents recommend daily intakes of 300-500 g of vegetables and 200-350 g of fruit for adults ${ }^{(11)}$. Despite the benefits described above, much of the Chinese population does not meet these recommended F \& V intake levels. According to a 2015 report on nutrition and chronic disease in China, Chinese people consumed an average of $269.4 \mathrm{~g}$ of fresh vegetables and $40 \cdot 7 \mathrm{~g}$ of fruit per $\mathrm{d}$ in $2012^{(12)}$.

Research has shown that F \& V consumption is linked to other behavioural factors and to individuals' sociodemographic milieu. F \& V consumption has been associated with education, income, age, alcohol consumption and smoking ${ }^{(13-16)}$. One study found differences by socioeconomic status and gender in $\mathrm{F} \& \mathrm{~V}$ intake in the $\mathrm{USA}^{(13)}$. Azagba and Sharaf found lower F \& V intake in Canada among adult men compared with women and among smokers compared with non-smokers ${ }^{(14)}$. An analysis from the USA showed that adults consuming at least five daily servings of $\mathrm{F} \& \mathrm{~V}$ were more likely to engage in at least moderate physical activity ${ }^{(17)}$. Disparities in F \& V intake by sociodemographic and behavioural factors are avoidable, 
and identification of factors associated with $\mathrm{F} \& \mathrm{~V}$ consumption is crucial for increasing F \& V intakes. In China, most studies in this area have focused on associations between $\mathrm{F} \& \mathrm{~V}$ intake and disease in the general population, but evidence is limited for disadvantaged populations. In this study, we measure associations of sociodemographic and behavioural characteristics with $\mathrm{F} \& \mathrm{~V}$ consumption in Chinese adults.

\section{Materials and methods}

\section{China Health and Nutrition Survey and study sample}

Data used for the study were from the 2015 wave of an ongoing longitudinal survey, the China Health and Nutrition Survey (CHNS), conducted by the Chinese Centers for Disease Control and the University of North Carolina at Chapel Hill. The survey was designed as a cohort study using multiple stratified cluster random sampling, with ten survey waves carried out between 1989 and 2015. For the 2015 wave, the survey was conducted in twelve provinces (Guangxi, Guizhou, Heilongjiang, Henan, Hubei, Hunan, Jiangsu, Liaoning, Shanxi, Shandong, Yunnan and Zhejiang) and three autonomous cities (Beijing, Chongqing and Shanghai). These twelve provinces and three autonomous cities contained approximately 59.5\% of the population of China.

Sociodemographic factors were collected in 2015 by household survey, individual survey, community survey, dietary measurement and physical examination. Questionnaires were administered by trained field interviewers. The CHNS questionnaire has been used in multiple waves of the study and has been shown to accurately capture usual diet intake. Detailed information on data collection is available in previous publications ${ }^{(18)}$. Diet was assessed by 24-h recalls for three consecutive days and a food inventory. For the food inventory, all food and edible oils, sugar and salt were measured with digital scales every day at the household level. Total household food consumption was estimated by the change in food inventory and waste. Adults were asked to describe all food and beverages consumed over three consecutive days, including food names, amount of food, type of meal and place of consumption. The amounts of individual foods consumed were estimated from the household inventory and the self-reported portion of the dish eaten at home. For the present analysis, total vegetable intake was calculated as the sum of all fresh vegetables eaten, including root vegetables, leguminous vegetables and sprouts, cucurbitaceous and solanaceous vegetables, allium vegetables, stems, leafy and flowering vegetables, aquatic vegetables, tubers and wild vegetables. Total fruit intake was calculated as the sum of all fresh fruits eaten, including kernel fruits, drupe (stone) fruits, berries, citrus fruits, tropical fruits and melons.
The CHNS was conducted in accordance with the Declaration of Helsinki, and the protocol was reviewed and approved by the Institutional Review Boards at the University of North Carolina at Chapel Hill and the National Institute for Nutrition and Health, Chinese Center for Disease Control and Prevention (No. 2015017, 18 August 2015). No separate ethics approval was required for this secondary analysis of data from the CHNS.

\section{Outcome variables}

The average daily consumption of fresh vegetables and fruits for each adult was calculated as the total amount of fresh vegetables or fruits consumed in $3 \mathrm{~d}$ divided by three. We examined the proportion of adults whose average daily fruit or vegetable consumption was equal to or above the cut-offs of $80 \mathrm{~g}$ of fruit (one serving) and $320 \mathrm{~g}$ of vegetables (four servings). These cut-offs were based on recommendations of the WHO, the World Cancer Research Fund and the 2016 Dietary Guidelines for Chinese Residents ${ }^{(1,19)}$. Fruit consumption was much lower than the Chinese recommendation. Accordingly, cut-offs were chosen in order to meet the WHO recommendations and to be as close as possible to the Chinese recommendation.

\section{Sociodemographic and behavioural variables}

Sociodemographic variables included age, sex, education level, geographic region in China (north or south), type of residence area (city, suburb, town or village, according to administrative divisions) and annual household income. Age was categorised as 18-34, 35-49 or 50-64 years old. Education was categorised as low ( $\leq 6$ years education), middle ( $7-12$ years education) or high ( $\geq 13$ years education). Personal annual income was collapsed into tertiles (low, moderate and high).

Behavioural variables examined included occupational physical activity, smoking status and alcohol consumption. Participants were asked to select their occupational physical activity level from a list of options including (1) very light physical activity (working in a sitting position, e.g. office worker, watch repairer), (2) light physical activity (working in standing position, e.g. salesperson, laboratory technician and teacher), (3) moderate physical activity (e.g. student, driver, electrician and metal worker), (4) heavy physical activity (e.g. farmer, dancer, steel worker and athlete) and (5) very heavy physical activity (e.g. loader, logger, miner and stonecutter). Level of occupational physical activity was assessed based on this question and was categorised as highly active (including heavy physical activity and very heavy physical activity), active (moderate physical activity), low activity (light physical activity) and inactive (very light physical activity). Smoking status was categorised as non-smoker, former smoker and current smoker. Alcohol consumption was categorised as non-drinker and drinker ('drinker' included both current and former drinkers). 


\section{Statistical analysis}

$\mathrm{F} \& \mathrm{~V}$ intake is expressed as the median with interquartile range $\left(25^{\text {th }}\right.$ to $75^{\text {th }}$ percentiles) due to skewed distributions. Bivariate analysis was used to examine relationship between the consumption of at least $80 \mathrm{~g}$ of fruit or $320 \mathrm{~g}$ of vegetables and sociodemographic, behavioural and physiological variables using $\chi^{2}$ tests. While the proportion of adults consuming at least $80 \mathrm{~g}$ of fruit per $\mathrm{d}$ and those consuming $320 \mathrm{~g}$ of vegetables per $\mathrm{d}$ are of concern from a public health standpoint, the outcomes were not statistically rare. Accordingly, we used log binomial regression models to estimate crude and adjusted risk ratios (RR) and $95 \%$ CI for associations between explanatory, confounding and outcome variables. CI that did not span 1 indicated statistical significance. All independent variables yielding statistically significant associations with $\mathrm{F} \& \mathrm{~V}$ intake in bivariate analyses were included in the log binomial regression models. All statistical analyses for the current study, including calculation of F \& V intake, were performed using Stata/sE version 14.0. $P$ values reported are two-tailed, and statistical significance was defined as $P<0.05$.

\section{Results}

The 2015 CHNS included data for 12226 adults aged 18-64 years. We excluded adults with incomplete or missing data on education ( $n$ 35), occupational activity (3), income (277) and smoking (1). This left a sample of 11910 adults aged 18-64 years included in the present analysis. The distribution of participants across sociodemographic and behavioural variables is shown in Table 1 . There were slightly more women than men, and the majority of participants had a low or medium educational level. Participants predominantly resided in villages, and roughly $60 \%$ lived in the southern region of China. There was a range of occupational activity levels reported; one-quarter of the sample were current smokers and $30 \%$ were current or former drinkers.

The most frequent vegetable source was stems, leafy and flowering vegetables, followed by cucurbitaceous and solanaceous vegetables, root vegetables, leguminous vegetables and sprouts, allium vegetables, aquatic vegetables, tuber and wild vegetables. The most frequent fruit source was kernel fruits, followed by citrus fruits, tropical fruits, berries, drupe (stone) fruits and melons.

\section{Bivariate analysis}

The overall median fruit and vegetable intake levels in the study were 0.00 (interquartile range: 0.00-65.72) and 245.66 (interquartile range: 163.48-352.56) g/d, respectively. Fruit intake of zero was common, with the exception of adults with high education or income levels and those living in a city. The right-hand side of Table 1 shows the proportion of adults consuming at least $80 \mathrm{~g} v$. $<80 \mathrm{~g}$ of fruit per $\mathrm{d}$ and those consuming at least $320 \mathrm{~g} v$. $<320 \mathrm{~g}$ of vegetables per d. Overall, $21 \cdot 1 \%$ of adults consumed at last $80 \mathrm{~g}$ of fruits per $\mathrm{d}$ and $31.6 \%$ consumed at least $320 \mathrm{~g}$ of vegetables per $\mathrm{d}$. The proportion of adults consuming at least $80 \mathrm{~g}$ of fruit per $\mathrm{d}$ and those consuming $320 \mathrm{~g}$ of vegetables per d varied significantly in relation to all sociodemographic and behavioural characteristics studied with the exception of personal income, for which significant variation was seen only for fruit consumption. F \& V consumption was somewhat higher among older participants. Women consumed more fruits, whereas men consumed more vegetables. Fruit consumption was directly proportional to education level, whereas vegetable consumption was lower in those with higher education levels. The highest fruit consumption levels were found among adults living in cities, whereas the highest vegetable consumption levels were among those living in the southern region. Fruit consumption was higher in the northern region, whereas vegetable consumption was higher in the southern region. Vegetable consumption was highest in adults with high occupational activity levels, whereas fruit consumption varied inversely with occupational activity. Fruit consumption was lowest among current smokers and current and former drinkers, whereas vegetable consumption was higher in current and former smokers and in drinkers than in non-smokers and non-drinkers. Finally, fruit consumption was directly proportional to personal income, whereas vegetable consumption did not vary significantly by income.

\section{Log binomial regression}

Table 2 displays crude and adjusted RR with 95\% confidence intervals (CI) for associations of sociodemographic and behavioural characteristics with fruit intake. Adults consuming at least $80 \mathrm{~g}$ of fruit were more likely to be those aged 50-64 years (RR: 1.20; $95 \%$ CI: 1.09, 1.33), women (RR: 1.34; $95 \%$ CI: 1.22, 1.46), urban residents (the reference group; statistically significant RR for all other areas of residence ranging from 0.66 to 0.85 ), those in the northern region (reference group; RR for southern region: 0.85; 95\% CI: 0.79, 0.91), those who were physically inactive (reference group; statistically significant RR for other groups ranging from 0.73 to 0.82 ), those with medium (RR: 1.28 ; $95 \%$ CI: $1.16,1.41$ ) or high income (RR: 1.58 ; $95 \%$ CI: $1.43,1.74$ ) levels and non-smokers (reference group; RR for current smokers: 0.86; $95 \%$ CI: 0.77, 0.96). Table 3 shows results of log binomial regression for vegetable consumption. Adults consuming at least $320 \mathrm{~g}$ of vegetable daily were more likely to be those aged 35 years and up (statistically significant RR ranging from 1.13 to 1.22), men (RR for women: 0.90; $95 \%$ CI: 0.84, 0.95), suburban residents (RR: 1.29; $95 \%$ CI: 1.19, 1.40), those in the southern region (RR: 1.88; $95 \% \mathrm{CI}: 1.76,2.01$ ), those with active (RR: $1 \cdot 11$; $95 \%$ CI: $1 \cdot 03,1 \cdot 21$ ) or high 
Table 1 Fruit and vegetable intake in relation to sociodemographic and behavioural variables, adults aged 18-64 years, China Health and Nutrition Survey 2015

\begin{tabular}{|c|c|c|c|c|c|c|c|c|c|c|c|}
\hline & \multirow[b]{3}{*}{$n$} & \multicolumn{4}{|c|}{ Intake in $\mathrm{g} / \mathrm{d}$} & \multicolumn{6}{|c|}{ Category of $F \& V$ intake } \\
\hline & & \multicolumn{2}{|c|}{ Fruit } & \multicolumn{2}{|c|}{ Vegetable } & \multicolumn{3}{|c|}{ Fruit } & \multicolumn{3}{|c|}{ Vegetable } \\
\hline & & Median & IQR & Median & IQR & $<80 \mathrm{~g} / \mathrm{d}$ & $\geq 80 \mathrm{~g} / \mathrm{d}$ & $P$ & $<320 \mathrm{~g} / \mathrm{d}$ & $\geq 320 \mathrm{~g} / \mathrm{d}$ & $P$ \\
\hline Age & & & & & & & & 0.032 & & & $<0.001$ \\
\hline $18-34$ years & 2315 & 0 & $0,65.00$ & 231.40 & $150 \cdot 01,333.33$ & 1853 & 462 & & 1668 & 647 & \\
\hline $35-49$ years & 4354 & 0 & $0,61.64$ & $242 \cdot 67$ & $161 \cdot 77,351 \cdot 30$ & 3463 & 891 & & 2982 & 1372 & \\
\hline $50-64$ years & 5241 & 0 & $0,66 \cdot 67$ & 252.89 & $168.40,359.87$ & 4076 & 1165 & & 3497 & 1744 & \\
\hline Sex & & & & & & & & $<0.001$ & & & $<0.001$ \\
\hline Male & 5555 & 0 & $0,47 \cdot 75$ & 256.75 & $171 \cdot 00,366 \cdot 67$ & 4609 & 946 & & 3610 & 1945 & \\
\hline Female & 6355 & 0 & $0,78.53$ & $235 \cdot 32$ & $156 \cdot 67,337.47$ & 4783 & 1572 & & 4537 & 1818 & \\
\hline Education & & & & & & & & $<0.001$ & & & $<0.001$ \\
\hline Low & 2774 & 0 & $0,35.03$ & 254.91 & $169.43,361.59$ & 2394 & 380 & & 1843 & 931 & \\
\hline Medium & 7523 & 0 & $0,66 \cdot 67$ & 249.01 & $164 \cdot 51,355 \cdot 70$ & 5881 & 1642 & & 5085 & 2438 & \\
\hline High & 1613 & $29 \cdot 50$ & $0,100 \cdot 40$ & $220 \cdot 01$ & $149 \cdot 19,316 \cdot 49$ & 1117 & 496 & & 1219 & 394 & \\
\hline Area of residence & & & & & & & & $<0.001$ & & & $<0.001$ \\
\hline City & 2522 & 33.33 & $0,109 \cdot 66$ & $230 \cdot 00$ & $151 \cdot 37,334.75$ & 1668 & 854 & & 1810 & 712 & \\
\hline Suburb & 2005 & 0 & $0,66 \cdot 45$ & $270 \cdot 67$ & $180 \cdot 62,407.90$ & 1572 & 433 & & 1226 & 779 & \\
\hline Town & 2155 & 0 & $0,66.00$ & 229.99 & $155 \cdot 20,331 \cdot 30$ & 1694 & 461 & & 1567 & 588 & \\
\hline Village & 5228 & 0 & $0,41.00$ & 251.69 & $167 \cdot 33,352 \cdot 66$ & 4458 & 770 & & 3544 & 1684 & \\
\hline Region & & & & & & & & $<0.001$ & & & $<0.001$ \\
\hline North & 4540 & 0 & $0,74.06$ & $200 \cdot 00$ & $131 \cdot 13,294 \cdot 40$ & 3449 & 1091 & & 3617 & 923 & \\
\hline South & 7370 & 0 & $0,56 \cdot 67$ & 274.51 & $190 \cdot 23,382 \cdot 88$ & 5943 & 1427 & & 4530 & 2840 & \\
\hline Occupational activity & & & & & & & & $<0.001$ & & & $<0.001$ \\
\hline Inactive & 3463 & 0 & $0,92 \cdot 36$ & 232.39 & $155 \cdot 95,337.05$ & 2482 & 981 & & 2483 & 980 & \\
\hline Low activity level & 3611 & 0 & $0,73.33$ & 238.32 & $162.33,339.99$ & 2762 & 849 & & 2556 & 1055 & \\
\hline Active & 2221 & 0 & $0,49.19$ & $253 \cdot 34$ & $168.27,360.01$ & 1861 & 360 & & 1466 & 755 & \\
\hline Highly active & 2615 & 0 & $0,25 \cdot 33$ & 271.46 & $172 \cdot 17,375 \cdot 59$ & 2287 & 328 & & 1642 & 973 & \\
\hline Smoking & & & & & & & & $<0.001$ & & & $<0.001$ \\
\hline Non-smoker & 8680 & 0 & $0,72 \cdot 47$ & $240 \cdot 00$ & $160 \cdot 00,343 \cdot 34$ & 6658 & 2022 & & 6098 & 2582 & \\
\hline Current smoker & 2989 & 0 & $0,38.00$ & 262.43 & $173.34,376 \cdot 67$ & 2544 & 445 & & 1893 & 1096 & \\
\hline Former smoker & 241 & 0 & $0,65.09$ & $266 \cdot 67$ & $186 \cdot 27,363 \cdot 79$ & 190 & 51 & & 156 & 85 & \\
\hline Drinking & & & & & & & & $<0.001$ & & & $<0.001$ \\
\hline Non-drinker & 8357 & 0 & $0,67.59$ & $230 \cdot 00$ & $151 \cdot 37,334 \cdot 75$ & 6497 & 1860 & & 5872 & 2485 & \\
\hline Drinker & 3524 & 0 & $0,52 \cdot 97$ & $260 \cdot 01$ & $171.66,372.50$ & 2876 & 648 & & 2254 & 1270 & \\
\hline Income tertile & & & & & & & & $<0.001$ & & & 0.288 \\
\hline Low & 3966 & 0 & $0,38.00$ & $250 \cdot 00$ & $160 \cdot 88,358 \cdot 75$ & 3409 & 557 & & 2683 & 1283 & \\
\hline Medium & 3969 & 0 & $0,62 \cdot 87$ & $243 \cdot 00$ & $161 \cdot 98,352 \cdot 63$ & 3153 & 816 & & 2710 & 1259 & \\
\hline High & 3975 & 8.21 & $0,93.33$ & $243 \cdot 32$ & $166 \cdot 67,348.67$ & 2830 & 1145 & & 2754 & 1221 & \\
\hline
\end{tabular}

$F \& V$, fruits and vegetables; IQR, interquartile range.

Differences in the proportion of adults consuming at least $80 \mathrm{~g}$ of fruit or $320 \mathrm{~g}$ of vegetables were compared using the $\chi^{2}$ test.

(RR: $1 \cdot 22 ; 95 \%$ CI:1.12, 1.32) occupational activity levels and current or former drinkers (RR: 1.11; $95 \% \mathrm{CI}: 1 \cdot 04,1 \cdot 18)$.

\section{Discussion}

In this study of adults in China, we found that only about one-fifth of adults met the recommended fruit consumption levels, and about one-third met the recommended level for vegetable consumption. We also found associations between numerous sociodemographic and behavioural characteristics and $\mathrm{F} \& \mathrm{~V}$ intake. Fruit consumption was lower among males, younger adults, those with lower incomes and education levels, those living in villages and in southern China, smokers and those with higher occupational activity levels. Vegetable consumption was found to be lower among younger adults, women, adults living in cities and in northern China, those with lower occupational physical activity levels and non-drinkers.

Results from our study are consistent with previous findings from Canada and the USA showing that people with higher education and income levels had significantly higher fruit intake $\mathrm{e}^{(14,20,21)}$. Findings in our study regarding fruit intake may be due in part to the cost of fruit in China, which is high. In addition, education level may be associated with fruit consumption through dietary knowledge. In contrast, we did not find a significant relationship between income and vegetable intake. This result may reflect the important role played in Chinese diets by vegetables, which are commonly used in dishes at every meal. In ancient China, it was recommended that diet should be close to nature, and vegetables were thought of as being preferable to meat. In winter, Chinese cabbage remains one of the most frequently consumed dishes in northern 
Factors related to fruit and vegetable intake

Table 2 Log binomial regression results, factors associated with fruit intake of at least $80 \mathrm{~g} / \mathrm{d}$ among adults aged 18-64 years, China Health and Nutrition Survey 2015

\begin{tabular}{|c|c|c|c|c|}
\hline & Crude RR & $95 \% \mathrm{Cl}$ & Adjusted RR & $95 \% \mathrm{Cl}$ \\
\hline \multicolumn{5}{|l|}{ Age } \\
\hline 18-34 years* & 1 & & 1 & \\
\hline $35-49$ years & 1.03 & $0.93,1.13$ & 1.09 & $0.99,1.20$ \\
\hline 50-64 years & $1 \cdot 11$ & $1 \cdot 01,1 \cdot 23$ & 1.20 & $1.09,1.33$ \\
\hline \multicolumn{5}{|l|}{ Sex } \\
\hline Male* $^{*}$ & 1 & & 1 & \\
\hline Female & 1.45 & $1.35,1.56$ & 1.34 & $1.22,1.46$ \\
\hline \multicolumn{5}{|l|}{ Education } \\
\hline Low $^{*}$ & 1 & & 1 & \\
\hline Medium & 1.59 & $1.44,1 \cdot 77$ & 1.39 & $1.25,1.55$ \\
\hline High & $2 \cdot 24$ & $1.99,2.53$ & 1.50 & $1.30,1.73$ \\
\hline \multicolumn{5}{|l|}{ Area of residence } \\
\hline City* & 1 & & 1 & \\
\hline Suburb & 0.64 & $0.58,0.70$ & 0.85 & $0.77,0.94$ \\
\hline Town & 0.63 & $0.57,0.70$ & 0.75 & $0.68,0.82$ \\
\hline Village & 0.43 & $0.40,0.47$ & 0.66 & $0.60,0.73$ \\
\hline \multicolumn{5}{|l|}{ Region } \\
\hline North* & 1 & & 1 & \\
\hline South & 0.81 & $0.75,0.86$ & 0.85 & $0.79,0.91$ \\
\hline \multicolumn{5}{|c|}{ Occupational physical activity } \\
\hline Inactive* & 1 & & 1 & \\
\hline Low activity level & 0.83 & $0.77,0.90$ & 0.96 & $0.89,1.04$ \\
\hline Active & 0.57 & $0.51,0.64$ & 0.82 & $0.73,0.91$ \\
\hline Highly active & 0.44 & $0.39,0.50$ & 0.73 & $0.64,0.83$ \\
\hline \multicolumn{5}{|l|}{ Income } \\
\hline Low $^{*}$ & 1 & & 1 & \\
\hline Medium & 1.46 & $1.33,1.62$ & 1.28 & $1.16,1.41$ \\
\hline High & 2.05 & $1 \cdot 87,2 \cdot 25$ & 1.58 & $1.43,1.74$ \\
\hline \multicolumn{5}{|l|}{ Smoking } \\
\hline Non-smoker* & 1 & & 1 & \\
\hline Current smoker & 0.64 & $0.58,0.70$ & 0.86 & $0.77,0.96$ \\
\hline Former smoker & 0.91 & $0.71,1.16$ & 1.04 & $0.82,1.34$ \\
\hline
\end{tabular}

$\mathrm{RR}$, risk ratio.

${ }^{\star}$ Reference category.

China today, and it is used in dozens of dishes in this region. Cultural factors and traditions have been found to be significantly related to $\mathrm{F} \& \mathrm{~V}$ consumption ${ }^{(14,15)}$. Despite the lack of an observed economic gradient, only one-third of adults met vegetable intake recommendations. Accordingly, efforts are needed to increase vegetable consumption across the economic spectrum in China.

We found that men tended to have higher vegetable intakes than women, whereas fruit intakes were higher among women. Our results regarding gender and fruit intake concur with studies from Canada, Germany and the USA finding that females had higher fruit intakes than males ${ }^{(14,22,23)}$. However, our results regarding gender and vegetable intake contrast with the findings of these studies. The difference in results between our study and previous research regarding $\mathrm{F} \& \mathrm{~V}$ intake in relation to gender may be partly due to higher overall knowledge regarding $\mathrm{F} \& \mathrm{~V}$ consumption among women compared with men, a pattern that has been observed in China as well as in other countries ${ }^{(24,25)}$. Alternatively, the frequent consumption of fruit as a snack in China may lead to disparities in fruit intake between men and women, as gender differences in lifestyles may entail differing eating schedules and habits. With China's rapid socioeconomics changes, lifestyles and consumption patterns have greatly improved for women. In addition, it has been shown that women in China spend more than men on personal health and fitness ${ }^{(26)}$. Finally, women in China have higher levels of health literacy compared with men, including health knowledge, lifestyle, behaviours and health-related skills ${ }^{(27)}$.

Our study found that adults who live in southern China and those who live in suburbs, villages and town settings were less likely to consume the recommended amount of fruit. This finding is similar to those from the China Kadoorie Biobank project, in which adults living in cities had higher fruit intakes than those living in villages ${ }^{(28)}$. This pattern may stem in part from the cost and accessibility of fruit ${ }^{(29)}$. Adults living in suburbs, villages and town areas tend to be poorer than those in cities and are served by fewer stores offering lower-cost foods ${ }^{(30)}$. Our findings regarding fruit consumption in relation to area of residence point to a need for targeted interventions to improve fruit intake in disadvantaged groups in China, especially individuals living in villages. The climate in southern China is generally warm and humid, with long periods of 
Table 3 Log binomial regression results, factors associated with vegetable intake of at least $320 \mathrm{~g} / \mathrm{d}$ among adults aged 18-64 years, China Health and Nutrition Survey 2015

\begin{tabular}{|c|c|c|c|c|}
\hline & Crude RR & $95 \% \mathrm{Cl}$ & Adjusted RR & $95 \% \mathrm{Cl}$ \\
\hline \multicolumn{5}{|l|}{ Age } \\
\hline $18-34$ years $^{*}$ & 1 & & 1 & \\
\hline $35-49$ years & $1 \cdot 13$ & $1.04,1.22$ & 1.13 & $1.05,1.22$ \\
\hline 50-64 years & $1 \cdot 19$ & $1 \cdot 10,1 \cdot 28$ & 1.22 & $1.13,1.31$ \\
\hline \multicolumn{5}{|l|}{ Sex } \\
\hline Male $^{\star}$ & 1 & & 1 & \\
\hline Female & 0.82 & $0.77,0.86$ & 0.90 & $0.84,0.95$ \\
\hline \multicolumn{5}{|l|}{ Area of residence } \\
\hline City* & 1 & & 1 & \\
\hline Suburb & 1.38 & $1.27,1.50$ & 1.29 & $1.19,1.40$ \\
\hline Town & 0.96 & $0.88,1.06$ & 0.95 & $0.87,1.05$ \\
\hline Village & $1 \cdot 14$ & $1 \cdot 06,1 \cdot 22$ & 1.05 & $0.97,1.14$ \\
\hline \multicolumn{5}{|l|}{ Region } \\
\hline North* & 1 & & 1 & \\
\hline South & 1.90 & $1 \cdot 78,2 \cdot 02$ & 1.88 & $1 \cdot 76,2 \cdot 01$ \\
\hline \multicolumn{5}{|c|}{ Occupational physical activity } \\
\hline Inactive $^{*}$ & 1 & & 1 & \\
\hline Low activity level & 1.03 & $0.96,1.11$ & 1.02 & $0.95,1.10$ \\
\hline Active & 1.20 & $1 \cdot 11,1 \cdot 30$ & $1 \cdot 11$ & $1.03,1.21$ \\
\hline Highly active & 1.31 & $1.22,1.41$ & $1 \cdot 22$ & $1.12,1.32$ \\
\hline \multicolumn{5}{|l|}{ Drinking } \\
\hline Non-drinker ${ }^{*}$ & 1 & & 1 & \\
\hline Drinker & $1 \cdot 21$ & $1 \cdot 15,1 \cdot 28$ & $1 \cdot 11$ & $1 \cdot 04,1 \cdot 18$ \\
\hline
\end{tabular}

sunshine, and is thus especially suitable for planting $\mathrm{F} \& \mathrm{~V}$. In contrast, the climate in the north is characterised by four distinct seasons, with high temperatures and rain in the summer and cold and dry conditions in the winter. The south is thus more suitable for growing $\mathrm{F} \& \mathrm{~V}$ than the north. Our study found factors associated with fruit intakes beyond natural conditions. It is not clear why we observed higher fruit intakes in the north yet higher vegetable intakes in the south. Accordingly, we recommend that future studies include more detailed exploration of geographic variation in $\mathrm{F} \& \mathrm{~V}$ intake in China.

Our findings that adults in the youngest age group (18-34 years old) consumed the lowest levels of fruits and vegetables are in agreement with previous reports from the USA and Australia(20,22,31), suggesting that elderly adults may be able to make healthier food choices than younger adults. While our findings indicate a need to promote adequate fruit and vegetable intake to the youngest age group, the reasons for variation in diet by age are not clear. Young adults frequently experience more flux and change over time in their daily lives than do older adults, and this is likely to affect their overall diet quality ${ }^{(32)}$. We recommend that future studies explore dietary patterns in relation to other psychosocial risk factors and lifestyle changes in young adults.

Adults in our study with moderate to high levels of occupational physical activity consumed less fruit but more vegetables than those with lower occupational activity levels. Our measurement of occupational physical activity was centred on activities during working hours, grouped by occupation. Accordingly, our finding of lower fruit consumption among those with lower occupational physical activity levels may partly reflect residual confounding from income and other occupation-related factors ${ }^{(15)}$. One study from the USA found that adults living in rural areas who engaged in at least moderate physical activity were more likely to consume five servings of $\mathrm{F} \& \mathrm{~V}$, echoing our findings for vegetable intake but not fruit intake ${ }^{(17)}$. Consistent with other studies, we found that smokers consumed less fruit than non-smokers ${ }^{(8,33)}$. These results suggest that non-smokers may make healthier food choices than smokers. However, we also found that drinkers consumed more vegetables than non-drinkers, a result that is inconsistent with previous studies ${ }^{(34)}$, suggesting that certain patterns of vegetable consumption in relation to alcohol use may be specific to China. Additional research is required to understand factors influencing the relationship between alcohol intake and diet in China.

Finally, we found that more adults in China adhere to recommendations for vegetable intake than for fruit intake. Again, factors associated with vegetable intake show patterns that may be unique to China. Our findings suggest that further dietary policies and interventions are needed in China and that fruits and vegetables must be considered separately rather than simply focusing on overall diet quality.

Several limitations to our study must be acknowledged. First, the sample was taken from an ongoing cohort study that may not reflect the overall adult population in China. The CHNS sample was randomly selected using a sampling 
strategy aimed at capturing a range of economic and demographic circumstances ${ }^{(18)}$. The twelve provinces and three autonomous cities sampled for the 2015 CHNS contained approximately half the population of China. Furthermore, the proportions of adults meeting minimum $\mathrm{F} \& \mathrm{~V}$ intake recommendations were similar to those reported by China National Nutrition and Health Surveillance and the China National Nutrition and Health Survey ${ }^{(11,35)}$. Second, F \& V consumption patterns vary by season, and we did not account for seasonality in our analyses. This may have led to inaccurate estimation of overall $\mathrm{F} \& \mathrm{~V}$ intake, though it is not likely to have created spurious associations with sociodemographic and behavioural factors. Third, this study is cross-sectional and cannot assess causality, including reverse causality. We recommend that future studies of dietary patterns in China assess the effects on $\mathrm{F} \& \mathrm{~V}$ intake of geographic mobility, educational and economic opportunities, uptake of public health interventions related to smoking and alcohol consumption and changes in occupational activity levels.

\section{Conclusions}

In this study, based on data from a national survey in China, we found that age, sex, area of residence, region and occupational activity were all associated with $\mathrm{F} \& \mathrm{~V}$ intake. This study helps identify nutritional problems in relation to sociodemographic and behavioural factors and suggests that further nutrition intervention programmes for adults are needed in China.

\section{Acknowledgements}

Acknowledgements: This research uses data from the China Health and Nutrition Survey (CHNS). The authors thank the team at National Institute for Nutrition and Health, Chinese Center for Disease Control and Prevention, the Carolina Population Center, University of North Carolina at Chapel Hill and the fifteen provincial Center for Disease Control and Prevention. Financial support: The authors thank the National Institute for Nutrition and Health, the China Center for Disease Control and Prevention, the Carolina Population Center (P2C HD050924, T32 HD007168), the University of North Carolina at Chapel Hill, the NIH (R01-HD30880, DK056350, R24 HD050924 and R01-HD38700) and the NIH Fogarty International Center (D43 TW009077, D43 TW007709) for financial support for the CHNS data collection and analysis files from 1989 to 2015 and later surveys, and 2015 Chinese Nutrition Society (CNS) Nutrition Research Foundation-DSM Research Fund (No. CNS2015075B) for 2015 survey. Conflict of interest: None. Authorship: L.L. and B.Z. contributed to the initial design of the analysis; L.L. conducted the analysis and wrote the manuscript; Y.F.O.Y., H.J.W., F.F.H., Y.W., J.G.Z., C.S., W.W.D., X.F.J., H.R.J. and Z.H.W. contributed to collect the data and reviewed the manuscript and B.Z. revised the manuscript. Ethics of buman subject participation: This study was conducted according to the guidelines laid down in the Declaration of Helsinki, and all procedures involving research study participants were approved by the Institutional Review Board of the National Institute for Nutrition and Health (no. 2015017, 18 August 2015). Written informed consent was obtained from all participants.

\section{References}

1. World Health Organization (2003) Diet, nutrition, and the prevention of chronic disease. In Joint WHO/FAO Expert Consultation WHO Technical Report Series no 916. Geneva: WHO.

2. Aune D, Giovannucci E, Boffetta P et al. (2017) Fruit and vegetable intake and the risk of cardiovascular disease, total cancer and all-cause mortality-a systematic review and dose-response meta-analysis of prospective studies. Int $J$ Epidemiol 46, 1029-1056.

3. Riboli E \& Norat T (2003) Epidemiologic evidence of the protective effect of fruit and vegetables on cancer risk. Am J Clin Nutr 78, 559S-569S.

4. Hu FB, Manson JE, Stampfer MJ et al. (2001) Diet, lifestyle, and the risk of type 2 diabetes mellitus in women. $N$ Engl J Med 345, 790-797.

5. Joshipura KJ, Hu FB, Manson JE et al. (2001) The effect of fruit and vegetable intake on risk for coronary heart disease. Ann Intern Med 134, 1106-1114.

6. He FJ, Nowson CA \& MacGregor GA (2006) Fruit and vegetable consumption and stroke: meta-analysis of cohort studies. Lancet 367, 320-326.

7. Cooper AJ, Sharp SJ, Lentjes MA et al. (2012) A prospective study of the association between quantity and variety of fruit and vegetable intake and incident type 2 diabetes. Diabetes Care 35, 1293-1300.

8. Vieira AR, Abar L, Vingeliene S et al. (2016) Fruits, vegetables and lung cancer risk: a systematic review and meta-analysis. Ann Oncol 27, 81-96.

9. Miedema MD, Petrone A, Shikany JM et al. (2015) Association of fruit and v.egetable consumption during early adulthood with the prevalence of coronary artery calcium after 20 years of follow-up: the coronary artery risk development in young adults (CARDIA) study. Circulation 132, 1990-1998.

10. GBD 2017 Diet Collaborators (2019) Health effects of dietary risks in 195 countries, 1990-2017: a systematic analysis for the Global Burden of Disease Study 2017. Lancet 393, 1958-1972.

11. Chang JL \& Wang Y (2016) The Report of China National Nutrition and Health Survey (2010-2013). Beijing: Peking University Medical Press.

12. National Health and Family Planning Commission of the PRC (2015) 2014 Report on Chinese Residents' Chronic Diseases and Nutrition. http://www.chinadaily.com.cn/m/chinahealth/ 2015-06/15/content_21008408.htm (accessed September 2019).

13. Dubowitz T, Heron M, Bird CE et al. (2008) Neighborhood socioeconomic status and fruit and vegetable intake among 
whites, blacks, and Mexican Americans in the United States. Am J Clin Nutr 87, 1883-1891.

14. Azagba S \& Sharaf MF (2011) Disparities in the frequency of fruit and vegetable consumption by socio-demographic and lifestyle characteristics in Canada. Nutr J 10, 118.

15. Pollard J, Kirk SF \& Cade JE (2002) Factors affecting food choice in relation to fruit and vegetable intake: a review. Nutr Res Rev 15, 373-387.

16. Shimotsu ST, Jones-Webb RJ, Lytle LA et al. (2012) The relationships among socioeconomic status, fruit and vegetable intake, and alcohol consumption. Am J Health Promot 27, 21-28.

17. Lutfiyya MN, Chang LF \& Lipsky MS (2012) A cross-sectional study of US rural adults' consumption of fruits and vegetables: do they consume at least five servings daily? $B M C$ Public Health 12, 280.

18. Popkin BM, Du S, Zhai F et al. (2010) Cohort profile: the China Health and Nutrition Survey: monitoring and understanding socio-economic and health change in China, 1989-2011. Int J Epidemiol 39, 1435-1440.

19. Chinese Nutrition Society (2016) The Chinese Dietary Guideline. Beijing: People's Health Publishing House.

20. Drewnowski A \& Rehm CD (2015) Socioeconomic gradient in consumption of whole fruit and $100 \%$ fruit juice among US children and adults. Nutr J 14, 3 .

21. Rehm CD, Penalvo JL, Afshin A et al. (2016) Dietary intake among US adults, 1999-2012. JAMA 315, 2542-2553.

22. Lee-Kwan SH, Moore LV, Blanck HM et al. (2017) Disparities in state-specific adult fruit and v.egetable consumption: United States, 2015. MMWR Morb Mortal Wkly Rep 66, 1241-1247.

23. Heuer T, Krems C, Moon K et al. (2015) Food consumption of adults in Germany: results of the German National Nutrition Survey II based on diet history interviews. $\mathrm{Br} J$ Nutr 113, 1603-1614.

24. Hu H, Gou L, Shi C et al. (2018) Survey on awareness rate of healthy lifestyle among residents in Nanjing. Chin J Health Educ 33, 330-333.

25. Baker AH \& Wardle J (2003) Sex differences in fruit and vegetable intake in older adults. Appetite 40, 269-275.
26. Yu S (2014) Social gender and life style: a preliminary analysis based on the 3rd edition (2010) of women's social status data in Sichuan. J Chengdu Normal Univ 30, $1-7,12$.

27. Yinghua L, Qunan M, Qi S et al. (2015) The level of health literacy of Chinese residents in 2012. Chin J Health Educ 31, 99-103.

28. Chenxil Q, Canqing Y, Huaidon D et al. (2015) Differences in diet intake frequency of adults: findings from half a million people in 10 areas in China. Chin J Epidemiol 36, 911-916.

29. Rehm CD, Monsivais P \& Drewnowski A (2011) The quality and monetary value of diets consumed by adults in the United States. Am J Clin Nutr 94, 1333-1339.

30. Miller V, Yusuf S, Chow CK et al. (2016) Availability, affordability, and consumption of fruits and vegetables in 18 countries across income levels: findings from the Prospective Urban Rural Epidemiology (PURE) study. Lancet Glob Health 4, e695-e703.

31. Chapman K, Goldsbury D, Watson W et al. (2017) Exploring perceptions and beliefs about the cost of fruit and vegetables and whether they are barriers to higher consumption. Appetite 113, 310-319.

32. Nelson MC, Story M, Larson NI et al. (2008) Emerging adulthood and college-aged youth: an overlooked age for weight-related behavior change. Obesity (Silver Spring) 16, 2205-2211.

33. Affret A, Severi G, Dow C et al. (2018) Socio-economic factors associated with an increase in fruit and vegetable consumption: a 12-year study in women from the E3N-EPIC study. Public Health Nutr 21, 740-755.

34. Myint PK, Welch AA, Bingham SA et al. (2007) Fruit and vegetable consumption and self-reported functional health in men and women in the European Prospective Investigation into Cancer-Norfolk (EPIC-Norfolk): a population-based cross-sectional study. Public Health Nutr 10, 34-41.

35. He Y, Zhao L, Yu D et al. (2016) Consumption of fruits and vegetables in Chinese adults from 2010 to 2012. Zhonghua Yu Fang Yi Xue Za Zhi 50, 221-224. 